\title{
ON UNIQUENESS OF WEAK SOLUTION TO MIXED PROBLEM FOR INTEGRO-DIFFERENTIAL AGGREGATION EQUATION
}

\section{V.F. VIL'DANOVA}

Abstract. In a well-known paper by A. Bertozzi, D. Slepcev (2010), there was established the existence and uniqueness of solution to a mixed problem for the aggregation equation

$$
u_{t}-\Delta A(x, u)+\operatorname{div}(u \nabla K * u)=0
$$

describing the evolution of a colony of bacteria in a bounded convex domain $\Omega$. In this paper we prove the existence and uniqueness of the solution to a mixed problem for a more general equation

$$
\beta(x, u)_{t}=\operatorname{div}(\nabla A(x, u)-\beta(x, u) G(u))+f(x, u) .
$$

The term $f(x, u)$ in the equation models the processes of "birth-destruction" of bacteria. The class of integral operators $G(v)$ is wide enough and contains, in particular, the convolution operators $\nabla K * u$. The vector kernel $g(x, y)$ of the operator $G(u)$ can have singularities.

Proof of the uniqueness of the solution in the work by A. Bertozzi, D. Slepcev was based on the conservation of the mass $\int_{\Omega} u(x, t) d x=$ const of bacteria and employed the convexity of $\Omega$ and the properties of the convolution operator. The presence of the "inhomogeneity" $f(x, u)$ violates the mass conservation. The proof of uniqueness proposed in the paper is suitable for a nonuniform equation and does not use the convexity of $\Omega$.

Keywords: aggregation equation, integro-differential equation, global solution, uniqueness of solution.

Mathematics Subject Classification: 35K20, 35K55, 35K65

\section{INTRODUCTION}

In the last decade there appeared many works devoted to studying the aggregation equation

$$
u_{t}=\operatorname{div}(\nabla A(x, u)-u \nabla K * u), \quad K * u=\int_{\mathbb{R}^{n}} K(x-y) u(y, t) d y,
$$

where the kernel $K$ can have singularity of Newtonian potential kind, see [1] and the references therein. A detailed survey of the results of such studies would have been too lengthy and this is why we mention only pioneering works devoted to equation (1).

In work [2], a model of bacteria chemotaxis was suggested as the system of equations

$$
\begin{aligned}
& u_{t}=\operatorname{div}(\nabla u-u \nabla v), \quad x \in \mathbb{R}^{n}, \quad t>0, \\
& -\Delta v=u, \quad x \in \mathbb{R}^{n}, \quad t>0 .
\end{aligned}
$$

V.F. VIL'DANOVA, ON UNIQUENESS OF WEAK SOLUTION TO MIXED PROBLEM FOR INTEGRO-DIFFERENTIAL AGGREGATION EQUATION.

(C) VIL'DANOVA V.F. 2018.

Submitted April 19, 2018.

The reported study was funded by RFBR according to the research project no. 18-01-00428a. 
It is obvious that for a bounded function $v$, system (2) is reduced to a single equation of form (1).

In work [3], system (2) was called Smolukhovsky-Poisson system and was used for studying gravitational collapse of a cloud of self-gravitating particles. In work [4], the same system was called Chavanis-Sommeria-Robert model with a reference to work [5].

Let $\Omega$ be a bounded domain in the space $\mathbb{R}^{n}, n \geqslant 2$, with the boundary in the class $C^{1}$. In the cylindrical domain $D^{T}=\Omega \times(0, T)$ we consider the equation

$$
\beta(x, u)_{t}=\operatorname{div}(\nabla A(x, u)-\beta(x, u) G(u))+f(x, u)
$$

with initial and boundary conditions

$$
\begin{array}{ll}
u(x, 0)=u_{0}(x), \quad c m u_{0}(x) \geqslant 0, & x \in \Omega, \\
(\nabla A(x, u)-\beta(x, u) G(u)) \cdot \nu=0 & \text { on } \quad \partial \Omega \times(0, T),
\end{array}
$$

where $\nu$ is the outward unit normal. The integral operator

$$
G(v)=\left(G_{1}(v), G_{2}(v), \ldots, G_{n}(v)\right)
$$

is defined by the formulae

$$
G_{i}(v)=\int_{\Omega} g_{i}(x, y) b(v(y)) d y .
$$

The aim of the work is to prove the existence and uniqueness of the weak solution to problem (3) -(5) in the cylinder $D^{T}$ with the height determined by data of the problem.

In work [6], the existence and uniqueness of the weak solution to problem (1), (4) with the boundary condition

$$
(\nabla A(x, u)-u \nabla K * u) \cdot \nu=0 \quad \text { on } \quad \partial \Omega \times(0, T)
$$

was proved in the case, when the kernel $K$ belonged to $C^{2}\left(\mathbb{R}^{n}\right)$. In earlier work [7] there were established the existence and uniqueness of the solution to the same problem in the case $A=A(u) \in C^{1}[0, \infty]$ as the domain $\Omega$ was convex. But the proof of the existence in this work contained a gap. In work [7, there were also considered Cauchy problem and a problem with a periodic boundary condition.

In the papers the authors knows, the function $A(x, u)$ increases in $u$ and among the power functions $A(x, u)=u^{m}$ only the case $m \geqslant 1$ was treated. We observe that the change $v=u^{m}$ in equation (1) with $A(x, u)=u^{m}$ leads us to equation (3) and this allows us to prove the existence of solution in the case $m \in(0,1)$, see Theorem 3 in Section 2 implied by the results in [8].

In work [8] there was prove the existence of a weak solution to the mixed problem in $D^{T}$ for the equation

$$
\beta(x, u)_{t}=\operatorname{div}(a(x, u, \nabla u)-\beta(x, u) G(u))+f(x, u)
$$

with the initial condition (4) and the boundary condition

$$
(a(x, u, \nabla u)-\beta(x, u) G(u)) \cdot \nu=0 \quad \text { on } \quad \partial \Omega \times(0, T) .
$$

The height $T$ of the cylinder $D^{T}$ is determined by the functions involved in the formulation of the problem. This result is discussed in details in Section 2.

The presence of a non-locality in equation (3) does not give a chance to employ the Kruzhkov's method of doubling variables applied in work [9] for proving the uniqueness of a renormalized solution to the mixed problem for the parabolic equation with a double nonlinearity.

A wider survey of works on aggregation equation can be found in work [6]. 


\section{MAIN RESUltS}

Suppose that $\beta(x, r), f(x, r), A(x, r)$ are Caratheodory functions. The function $A(x, u) \in$ $C^{1}(\bar{\Omega} \times[0, \infty)), A(x, u) \geqslant 0$, has a positive derivative $A_{u}$,

$$
\begin{aligned}
& A_{u}(x, u)>a_{0}>0, \quad x \in \Omega, \quad u>0 \\
& (A(x, u)-A(x, v))(\beta(x, u)-\beta(x, v)) \geqslant a_{0}(u-v)^{2} \quad \text { for all } \quad u, v \in \mathbb{R}_{+} .
\end{aligned}
$$

We describe the conditions for the functions involved in the integral operator $G(v)$ :

$$
g_{i}(x, y) \in C^{1}(P), \quad P=\{(x, y): x, y \in \bar{\Omega}, x \neq y\} .
$$

We assume that for some $\lambda<n$ the inequalities hold:

$$
\begin{aligned}
& \sum_{i=1}^{n}\left|\left(g_{i}(x, y)\right)_{x_{i}}\right|+\left|g_{i}(x, y)\right| \leqslant C\left(1+|x-y|^{-\lambda}\right), \quad(x, y) \in P, \\
& \sum_{i=1}^{n} \nu_{i} g_{i}(x, y) \leqslant 0, \quad x \in \partial \Omega, \quad y \in \Omega .
\end{aligned}
$$

The functions $f(x, s), b(s), f(x, 0)=0, b(0)=0$, satisfy the Lipschitz condition:

$$
\begin{array}{ll}
\left|f\left(x, s_{1}\right)-f\left(x, s_{2}\right)\right| \leqslant L_{r}\left|s_{1}-s_{2}\right|, & s_{1}, s_{2} \in[0, r] \quad \text { for all } \quad r>0, \\
\left|b\left(s_{1}\right)-b\left(s_{2}\right)\right| \leqslant L_{k}\left|s_{1}-s_{2}\right|, & s_{1}, s_{2} \in[0, k] \quad \text { for all } \quad k>0 .
\end{array}
$$

It follows from the condition $\lambda<n$ that there exists a number $\bar{q}>1$ such that $\lambda<\frac{n}{\bar{q}}$. We fix complex conjugate numbers $q, \bar{q}$ such that $\frac{1}{q}+\frac{1}{\bar{q}}=1$.

The weak solution is defined as follows.

Definition 1. The function $u: D^{T} \rightarrow[0, \infty), u \geqslant 0$, is called a weak solution to problem (3) - (5) if

$$
u \in L_{\infty}\left(D^{T}\right), \quad \beta(x, u) \in L_{\infty}\left(0, T ; L_{q}(\Omega)\right), \quad A(x, u) \in L_{2}\left(0, T ; H^{1}(\Omega)\right)
$$

and for all Lipschitz functions $\xi \in \operatorname{Lip}\left(\overline{D^{T}}\right)$ obeying $\xi(T)=0$ the identity holds:

$$
\int_{D^{T}}\left(-\beta(x, u) \xi_{t}+(\nabla A(x, u)-\beta(x, u) G(u)) \cdot \nabla \xi-f(x, u) \xi\right) d x d t=\int_{\Omega} u_{0}(x) \xi(x, 0) d x .
$$

Theorem 1. Assume that conditions (8) -13 hold and let there exists a non-negative solution to problem (3) - (5). Then this solution is unique.

We shall use the following statements on the estimates of the potential type integrals (see, for instance, [10, Ch. I, Sect. 6]).

Lemma 1. If

$$
\lambda<\frac{n}{\bar{q}}, \quad \frac{1}{q}+\frac{1}{\bar{q}}=1, \quad 1<q<\infty, \quad f(x) \in L_{q}(\Omega),
$$

then the function

$$
v(x)=\int_{\Omega} \frac{f(y) d y}{|x-y|^{\lambda}}
$$

is continuous in $\mathbb{R}^{n}$ and satisfies the inequality

$$
|v(x)| \leqslant C\|f\|_{q, \Omega} .
$$


Lemma 2. Assume that the domain $\Omega$ is bounded and $\lambda<n, f(x) \in L_{2}(\Omega)$. Then the function

$$
v(x)=\int_{\Omega} \frac{f(y) d y}{|x-y|^{\lambda}}
$$

is square summable and the inequality holds:

$$
\|v(x)\|_{2, \Omega} \leqslant C\|f\|_{2, \Omega} .
$$

Let $M_{0}=\left\|u_{0}\right\|_{L_{\infty}(\Omega)}$ and $M_{T}>M_{0}$ be an arbitrary number. We are going to obtain some estimate for the integral operator $G(v)$. We consider a measurable function $v(x)$; this function obeys $|v(x)| \leqslant M_{T}$. It follows from Lemma 1 and conditions (10), (13) that

$$
G(v) \in C^{1}\left(\mathbb{R}^{n}\right), \quad|G(v)(x)| \leqslant C_{G}, \quad|\nabla G(v)(x)| \leqslant d_{G}, \quad x \in \Omega .
$$

Let us provide a statement (see [8]) on existence of solution to problem (6), (4), (7).

In what follows, the nonlinearity exponents $p_{i}(x)$ satisfy the condition:

$$
\left|p_{i}(x)-p_{i}(y)\right| \leqslant \frac{C}{-\ln |x-y|}, \quad i=\overline{1, n},
$$

as $|x-y| \leqslant \frac{1}{2}, x, y \in \bar{\Omega}$. In work [8], the conditions for the functions involved in equation (6) were as follows. The function $\beta(x, r)$ is odd in $r \in \mathbb{R}$ and for some $M_{0}, M_{T}$ it satisfies the conditions

$$
\begin{aligned}
& s \beta(x, r) \leqslant r \beta(x, s) \quad \text { as } \quad 0<M_{0} \leqslant r<s \leqslant M_{T}, \quad x \in \Omega ; \\
& \beta\left(x, M_{T}\right) \in L_{q}(\Omega), \quad \text { where } \quad q \geqslant \max _{j}\left(\bar{p}_{j}(x)\right), \quad x \in \Omega . \\
& |\nabla \beta(x, r)| \leqslant N_{g}|\beta(x, r)|, \quad r \in\left[0, M_{T}\right] \quad x \in \Omega .
\end{aligned}
$$

The function $q_{1}(x, r)$ is defined by the identity $f=\beta(x, r) q_{1}(x, r)$ and is bounded

$$
\left|q_{1}(x, r)\right| \leqslant q_{0} \quad \text { as } \quad|r| \leqslant M_{T} .
$$

The functions $a_{i}(x, r, y)$ are continuous in $r \in \mathbb{R}, y \in \mathbb{R}^{n}$ and measurable in $x \in \Omega$. There exist a function $F(x) \in L_{1}(\Omega)$ and a continuous function $C(m), m \geqslant 0$, such that

$$
\left|a_{j}(x, r, y)\right|^{\bar{p}_{j}(x)} \leqslant C(m)\left(F(x)+\sum_{i=1}^{n}\left|y_{i}\right|^{p_{i}(x)}\right),
$$

for all $r \in[-m, m], y \in \mathbb{R}^{n}, x \in \Omega$.

The monotonicity and coercivity conditions are introduced as

$$
\begin{aligned}
& (a(x, r, y)-a(x, r, z)) \cdot(y-z) \geqslant 0, \quad y \neq z ; \\
& a(x, r, y) \cdot y \geqslant \delta_{0} \sum_{i=1}^{n}\left|y_{i}\right|^{p_{i}(x)}-F(x), \quad \text { for all } \quad r \in \mathbb{R}, \quad y \in \mathbb{R}^{n}, \quad x \in \Omega .
\end{aligned}
$$

The function $B(x, r)$ is defined by the identity

$$
B(x, r)=\int_{0}^{r} s d_{s} \beta(x, s),
$$

grows in $r$ and obeys the inequality

$$
0 \leqslant B(x, r) \leqslant r \beta(x, r), \quad r \geqslant 0 .
$$

This is why $B\left(x, M_{T}\right) \in L_{q}(\Omega)$. 
Theorem 2. (see [8]). Assume that conditions (10), (11), (13), (16)-(23) hold and $0 \leqslant$ $u_{0}(x) \leqslant M_{0}$. Then there exists $T=T\left(M_{0}, M_{T}, q_{0}, d_{G}, N_{g}\right)$ and a weak solution to problem (6), (41), (7) such that $0 \leqslant u(x, t) \leqslant M_{T}$.

We do not provide here the definition of the weak solution to problem (6), (4), (7) since it is standard and for the considered in the work problem (3)-(5) it coincides with Definition 1.

The next statement follows Theorem 2 .

Theorem 3. Assume that conditions (8), (10), (11), (13), (17) $-(20)$ hold and $0 \leqslant u_{0}(x) \leqslant$ $M_{0}$. Then there exists a weak solution to problem (3) (5) in the cylinder $D^{T}$, where $T=$ $T\left(M_{0}, M_{T}, q_{0}, d_{G}, N_{g}\right)$.

It is obvious that problem (3)-(5) is a particular case of problem (6), (4), (7). This is why it is sufficient to confirm that the assumptions of Theorem 3 ensures the assumptions of Theorem 3. For equation (3), the functions $a_{j}$ are of the form $a_{j}=A_{u} u_{x_{j}}+A_{x_{j}}$, and this is why condition (21) holds as $p_{j}=2$ thanks to the smoothness of the function $A(x, u)$. Conditions (22) and (23) are also satisfied by (8) and the boundedness of $u$.

As an important example, in $D^{T}$ we consider the equation

$$
u_{t}=\operatorname{div}\left(\nabla u^{m}-u \nabla K * u\right), \quad m \in(0,1),
$$

where the kernel $K$ satisfies the condition

$$
\nabla_{x}^{2} K(x, y) \leqslant C\left(1+|x-y|^{-\lambda}\right), \quad(x, y) \in P, \quad \lambda<n .
$$

The change $v=u^{m}$ leads us to the equation

$$
\left(v^{\frac{1}{m}}\right)_{t}=\operatorname{div}\left(\nabla v-u \nabla K * v^{\frac{1}{m}}\right)
$$

of form (3). By Theorem 3 we establish that the corresponding problem possesses a weak solution as $m \in(0,1)$, but we do not state the uniqueness.

\section{Proof of Theorem 1 on uniqueness of SOlution}

Let us establish an auxiliary statement.

Lemma 3. Let functions $u(x, t), v(x, t)$ be weak solution to problem (3) -(5),

$$
N(t)=\int_{\Omega}|u(x, t)-v(x, t)| d x, \quad s(t)=\int_{\Omega}(\beta(x, u(x, t))-\beta(x, v(x, t))) d x .
$$

Then for all $\tau \in[0, T]$ we have:

$$
\int_{0}^{\tau} s^{2}(t) d t \leqslant L_{r}^{2} \tau^{2} \int_{0}^{\tau} N^{2}(t) d t
$$

and

$$
\int_{0}^{\tau} s^{2}(t) d t \leqslant C(\Omega) \tau^{2} \int_{0}^{\tau} \int_{\Omega}|u(x, t)-v(x, t)|^{2} d x d t
$$

where $r=\|u+v\|_{L_{\infty}\left(D^{T}\right)}, L_{r}$ is a constant in 12 . 
Proof. We write relation (14) for the function $v$, we deduct it from (14) and we obtain

$$
\begin{gathered}
\int_{0}^{T} \int_{\Omega}\left((\beta(x, v)-\beta(x, u)) \xi_{t}+(\nabla A(x, u)-\nabla A(x, v)+\beta(x, v) G(v)\right. \\
\quad-\beta(x, u) G(u)) \cdot \nabla \xi) d x d t=\int_{0}^{T} \int_{\Omega}(f(x, u)-f(x, v)) \xi d x d t .
\end{gathered}
$$

Substituting here $\xi=\xi(t) \in C_{0}^{\infty}(0, T)$, we obtain:

$$
\int_{0}^{T} \xi^{\prime}(t) \int_{\Omega}(\beta(x, u)-\beta(x, v)) d x d t=\int_{0}^{T} \xi(t) \int_{\Omega}(f(x, v)-f(x, u)) d x d t .
$$

This means that the function $s(t)$ is absolutely continuous in $t$. This is why, to complete the proof of the lemma, it is sufficient to substitute the function

$$
\xi(t)=\int_{t}^{\tau} s(r) d r, \quad t \in[0, \tau], \quad \xi(t)=0, \quad t>\tau,
$$

into (24). By (12) and Steklov-Friedrichs inequality we have:

$$
\int_{0}^{\tau} s^{2}(t) d t \leqslant \int_{0}^{\tau} L_{r} N(t)|\xi(t)| d t \leqslant L_{r} \tau\left(\int_{0}^{\tau} N^{2}(t) d t \int_{0}^{\tau} s^{2}(t) d t\right)^{\frac{1}{2}} .
$$

This implies easily the stated inequalities.

Proof of Theorem 1. Let $u$ and $v$ be solutions to problem (3)-(5). Given $t \in(0, T)$, we define the function $\phi(x, t)$ as the solution to the Neumann problem

$$
\Delta \phi(x, t)=\beta(x, u(x, t))-\beta(x, v(x, t))-\bar{s}(t), \quad x \in \Omega, \quad \frac{\partial \phi}{\partial \nu}=0, \quad x \in \partial \Omega,
$$

where $\bar{s}(t)=\frac{s(t)}{\text { meas } \Omega}$. The solvability condition of this problem is the orthogonality in $L_{2}(\Omega)$ of the right hand in this equation to the solutions of the homogeneous equation (see, for instance, [11, Ch. II, Thm. 5.2]). The solutions of the problem for the homogeneous equation are constant functions only. According Lemma 3, we have the identity

$$
\int_{\Omega}(\beta(x, u(x, t))-\beta(x, v(x, t))-\bar{s}(t)) d x=0,
$$

that is, the solvability condition of the Neumann problem holds. We can also assume that

$$
\int_{\Omega} \phi(x, t) d x=0
$$

We observe that $\phi(x, 0)=0$. Since

$$
(\beta(x, u(x, t))-\beta(x, v(x, t))) \in L_{\infty}\left(0, T ; L_{q}(\Omega)\right) \cap H^{1}\left(0, T ; H^{-1}(\Omega)\right),
$$

then

$$
\phi \in L_{\infty}\left(0, T ; H^{2}(\Omega)\right) \cap H^{1}\left(0, T ; H^{1}(\Omega)\right),
$$

see, for instance, [11, Ch. II, Eq. (5.4)]. Then $\nabla \phi \in C\left(0, T ; L_{2}(\Omega)\right)$ and the identity holds:

$$
-\int_{\Omega} \nabla \phi \cdot \nabla w d x=\int_{\Omega}(\beta(x, u(x, t))-\beta(x, v(x, t))-\bar{s}(t)) w(x) d x,
$$


for each $w \in W_{2}^{1}(\Omega)$.

Let us transform equation (24). We denote

$$
P(x, t)=\nabla A(x, u)-\nabla A(x, v)+\beta(x, v) G(v)-\beta(x, u) G(u) .
$$

It follows from Definition 1 that $P \in L_{2}\left(D^{T}\right)$. We let $F(x, t)=f(x, u)-f(x, v) \in L_{2}\left(D^{T}\right)$. We substitute

$$
\xi=\varphi_{-h}=\frac{1}{h} \int_{t-h}^{t} \varphi(x, r) d r
$$

into (24), where $\varphi \in C^{\infty}\left(\overline{D^{T}}\right), \varphi=0$ as $t \geqslant T-\delta$. Taking into consideration (26), we obtain:

$$
\int_{D^{T}}\left(\nabla \phi \cdot \nabla\left(\varphi_{-h}\right)_{t}-\bar{s}(t)\left(\varphi_{-h}\right)_{t}+P \cdot \nabla \varphi_{-h}-F \varphi_{-h}\right) d x d t=0 .
$$

Employing the properties of Steklov averaging, we rewrite this as

$$
\int_{D^{T}}\left(-\left(\nabla \phi_{h}\right)_{t} \cdot \nabla \varphi-\left(\bar{s}_{h}\right)_{t} \varphi+P_{h} \cdot \nabla \varphi-F_{h} \varphi\right) d x d t=0 .
$$

Let $\chi_{(0, \tau)}$ be the characteristic function of the interval $(0, \tau)$. We substitute $\varphi=\chi(0<t<\tau) \phi_{h}$ into the latter equation and we pass to the limit as $h \rightarrow 0$. We obtain:

$$
-\frac{1}{2} \int_{\Omega}|\nabla \phi(\tau)|^{2}+\int_{0}^{\tau} \int_{\Omega}\left(-\bar{s}_{t} \phi+P \cdot \nabla \phi-F \phi\right) d x d t=0 .
$$

Taking into consideration that

$$
\int_{\Omega} \phi(x, t) d x=0
$$

we get:

$$
\begin{aligned}
\frac{1}{2} \int_{\Omega}|\nabla \phi(\tau)|^{2} d x= & \int_{0}^{\tau} \int_{\Omega} \nabla(A(x, u)-A(x, v)) \cdot \nabla \phi d x d t \\
& -\int_{0}^{\tau} \int_{\Omega}(G(u) \beta(x, u)-G(v) \beta(x, v)) \cdot \nabla \phi d x d t \\
& -\int_{0}^{\tau} \int_{\Omega}(f(x, u)-f(x, v)) \phi d x d t=I_{1}+I_{2}+I_{3} .
\end{aligned}
$$

Since $A(x, u)-A(x, v) \in L_{2}\left(0, T, H^{1}(\Omega)\right)$ and the function $A$ increases in the second variable, employing (26) and Lemma 3 and taking into consideration (9), we can write the relations:

$$
\begin{aligned}
I_{1} & =-\int_{0}^{\tau} \int_{\Omega}(A(x, u)-A(x, v))(\beta(x, u)-\beta(x, v)-\bar{s}(t)) d x d t \\
& \leqslant-\int_{0}^{\tau} \int_{\Omega}\left(a_{0}(u-v)^{2}-A_{M}|(u-v) \bar{s}(t)| d x d t \leqslant \int_{0}^{\tau} \int_{\Omega}\left(C \tau-a_{0}\right)(u-v)^{2} d x d t .\right.
\end{aligned}
$$


We rewrite integral $I_{2}$ as

$$
\begin{aligned}
I_{2}= & -\int_{0}^{\tau} \int_{\Omega}(G(u) \beta(x, u)-G(v) \beta(x, v)) \cdot \nabla \phi d x d t=-\int_{0}^{\tau} \int_{\Omega}(\beta(x, u)-\beta(x, v)) G(u) \cdot \nabla \phi d x d t \\
& +\int_{0}^{\tau} \int_{\Omega} \beta(x, v)(G(u)-G(v)) \cdot \nabla \phi d x d t=I_{4}+I_{5} .
\end{aligned}
$$

Employing (26) for the first term, we obtain

$$
I_{4}=\int_{0}^{\tau} \int_{\Omega} \nabla \phi \cdot \nabla(G(u) \cdot \nabla \phi) d x d t-\int_{0}^{\tau} \int_{\Omega} \bar{s}(t) G(u) \cdot \nabla \phi d x d t=I_{41}+I_{42} .
$$

By Lemma 3 we have the estimate

$$
I_{42} \leqslant C \tau\|u-v\|_{L_{2}\left(D_{0}^{\tau}\right)}\|\nabla \phi\|_{L_{2}\left(D_{0}^{\tau}\right)}
$$

where $D_{0}^{\tau}=\Omega \times(0, \tau)$. Then,

$$
\begin{aligned}
I_{41}= & \int_{0}^{\tau} \int_{\Omega} \partial_{i} \phi(x) \partial_{j} \phi(x) \int_{\Omega} \partial_{i} g_{j}(x, y) b(u(y, t)) d y d x d t \\
& +\int_{0}^{\tau} \int_{\Omega} \partial_{i} \phi(x) \partial_{i} \partial_{j} \phi(x) \int_{\Omega} g_{j}(x, y) b(u(y, t)) d y d x d t=I_{6}+I_{7} .
\end{aligned}
$$

We apply Gauss-Ostrogradsky formula to the integral $I_{7}$ :

$$
\begin{aligned}
I_{7}= & -\int_{0}^{\tau} \int_{\Omega} \partial_{j} \partial_{i} \phi(x) \partial_{i} \phi(x) \int_{\Omega} g_{j}(x, y) b(u(y, t)) d y d x d t \\
& -\int_{0}^{\tau} \int_{\Omega} \partial_{i} \phi(x) \partial_{i} \phi(x) \int_{\Omega} \partial_{j} g_{j}(x, y) b(u(y, t)) d y d x d t \\
& +\int_{0}^{\tau} \int_{\partial \Omega} \partial_{i} \phi(x) \partial_{i} \phi(x) \nu_{j} \int_{\Omega} g_{j}(x, y) b(u(y, t)) d y d S d t .
\end{aligned}
$$

Then in view of (11) we obtain

$$
I_{7} \leqslant-\frac{1}{2} \int_{0}^{\tau} \int_{\Omega}|\nabla \phi|^{2} \int_{\Omega} \partial_{j} g_{j}(x, y) b(u(y, t)) d y d x d t .
$$

Employing conditions (10), 13), we estimate the integral:

$$
\begin{aligned}
\int_{\Omega}\left|\partial_{i} g_{j}(x, y) b(u(y, t))\right| d y & \leqslant \int_{\Omega} C\left(1+|x-y|^{-\lambda}\right)|b(u(y, t))| d y \\
& \leqslant C\left\|u_{0}\right\|_{L_{1}(\Omega)}+C \int_{\Omega} \frac{|b(u(y, t))|}{|x-y|^{\lambda}} d y \leqslant C\left(M_{T}\right) .
\end{aligned}
$$


We have

$$
I_{6} \leqslant C\left(M_{T}\right) \int_{0}^{\tau} \int_{\Omega}|\nabla \phi|^{2} d x d t
$$

We substitute the obtained estimates for $I_{6}$ and $I_{7}$ into $I_{4}$ :

$$
I_{4} \leqslant \int_{0}^{\tau} \int_{\Omega}\left(C|\nabla \phi|^{2}+\tau^{2}(u-v)^{2}\right) d x d t .
$$

Employing conditions (10), 13) and Lemma 2, we establish the inequalities:

$$
\left\|G_{j}(u(t))-G_{j}(v(t))\right\|_{L_{2}(\Omega)}<C(\Omega)\|u(t)-v(t)\|_{L_{2}(\Omega)}, \quad j=\overline{1, n} .
$$

Hence,

$$
I_{5} \leqslant C \int_{0}^{\tau}\|u(t)-v(t)\|_{L_{2}(\Omega)}\|\nabla \phi(t)\|_{L_{2}(\Omega)} d t .
$$

Then

$$
I_{5} \leqslant C(\tau) \int_{0}^{\tau}\|\nabla \phi(t)\|_{L_{2}(\Omega)}^{2} d t+\tau \int_{0}^{\tau} \int_{\Omega}(u-v)^{2} d x d t .
$$

Letting $\eta(t)=\left(\int_{\Omega}|\nabla \phi(t)|^{2} d x\right)^{\frac{1}{2}}$, by 27$)$ and previous estimates we obtain

$$
\begin{aligned}
\frac{1}{2} \eta^{2}(\tau) & +\left(a_{0}-C\left(\tau+\tau^{2}\right)\right) \int_{0}^{\tau} \int_{\Omega}(u-v)^{2} d x d t \\
& \leqslant C \int_{0}^{\tau} \eta^{2}(t) d t+\int_{0}^{\tau} \int_{\Omega}|(f(x, u)-f(x, v)) \phi| d x d t .
\end{aligned}
$$

Employing condition $(12)$ for the function $f(x, u)$ and Poincaré inequality, we establish that

$$
\begin{aligned}
\int_{0}^{\tau} \int_{\Omega}|f(x, u)-f(x, v)||\phi| d x d t & \leqslant \frac{a_{0}}{2} \int_{0}^{\tau} \int_{\Omega}(u-v)^{2} d x d t+\frac{L_{r}^{2}}{a_{0}} \int_{0}^{\tau} \int_{\Omega}|\phi|^{2} d x d t \\
& \leqslant \frac{a_{0}}{2} \int_{0}^{\tau} \int_{\Omega}(u-v)^{2} d x d t+\frac{C_{1} L_{r}^{2}}{a_{0}} \int_{0}^{\tau} \int_{\Omega}|\nabla \phi|^{2} d x d t .
\end{aligned}
$$

Hence, for sufficiently small $\tau$ it follows from (30), (31) that

$$
\eta^{2}(\tau) \leqslant C_{2} \int_{0}^{\tau} \eta^{2}(t) d t
$$

By means of Grönwall's lemma we hence conlcude that $\eta(t)=0$ for all $0 \leqslant t \leqslant \tau$. Therefore, $u \equiv v$ in the cylinder $\Omega \times(0, \tau)$. In the same way we establish the identity $u \equiv v$ in the cylinder $\Omega \times(\tau, 2 \tau)$ and so forth. The proof is complete. 


\section{BIBLIOGRAPHY}

1. J.A. Carrillo, S. Hittmeir, B. Volzone, Y. Yao. Nonlinear aggregation-diffusion equations: radial symmetry and long time asymptotics // Preprtin: arXiv:1603.07767v1, (2016).

2. E.F. Keller, L.A. Segel. Initiation of slide mold aggregation viewed as an instability // J. Theor. Biol. 26:3, 399-415 (1970).

3. P.H. Chavanis, C. Rosier, C. Sire. Thermodynamics of self-gravitating systems // Foundat. Phys. 33:2, 223-269 (2003).

4. P. Biler, T. Nadzieja. Global and exploding solutions in a model of self-gravitating systems // Rep. Math. Phys. 52:2, 205-225 (2003).

5. P.H. Chavanis, J. Sommeria and R. Robert. Statistical mechanics of two-dimensional vortices and collisionless stellar systems// J. Astrophys. 471:1, 385-399 (1996).

6. V.F. Vil'danova. Existence and uniqueness of a weak solution of a nonlocal aggregation equation with degenerate diffusion of general form // Matem. Sborn. 209:2, 66-81 (2018). [Sb. Math. 209:2, 206-221 (2018)].

7. A. Bertozzi, D. Slepcev. Existence and uniqueness of solutions to an aggregation equation with degenerate diffusion // Comm. Pure Appl. Anal. 9:6, 1617-1637 (2010).

8. V.F. Vildanova, F.Kh. Mukminov. Existence of weak solution of the aggregation integro-differential equation // Sovr. Probl. Matem. Fund. Napr. 63:4, 557-572 (2017).

9. Kh. Mukminov. Uniqueness of the renormalized solution of an elliptic-parabolic problem in anisotropic Sobolev-Orlicz spaces // Matem. Sborn. 208:8 106-125 (2017). [Sb. Math. 208:8, 11871206 (2017).]

10. S.L. Sobolev. Some applications of functional analysis in mathematical physics. Nauka, Moscow (1988). [Transl. Math. Monog. 90. AMS, Providence, RI (1991).]

11. J.L. Lions, E. Magenes. Non-homogeneous boundary value problems and applications. Vol. I. . Springer, Berlin (1972).

Venera Fidarisovna Vil'danova

Bashkir State Pedagogical University

named after M.Akhmulla

October rev. str. 3a,

450000, Ufa, Russia

E-mail: gilvenera@mail.ru 